\title{
Using Heterogeneous Wireless Sensor Networks in a Telemonitoring System for Healthcare
}

\author{
Juan M. Corchado, Javier Bajo, Dante I. Tapia, and Ajith Abraham
}

\begin{abstract}
Ambient intelligence has acquired great importance in recent years and requires the development of new innovative solutions. This paper presents a distributed telemonitoring system, aimed at improving healthcare and assistance to dependent people at their homes. The system implements a service-oriented architecture based platform, which allows heterogeneous wireless sensor networks to communicate in a distributed way independent of time and location restrictions. This approach provides the system with a higher ability to recover from errors and a better flexibility to change their behavior at execution time. Preliminary results are presented in this paper.
\end{abstract}

Index Terms-Ambient intelligence (AmI), healthcare, servicesoriented architectures (SOAs), wireless sensors networks (WSNs).

\section{INTRODUCTION}

$\mathbf{P}$ EOPLE are currently surrounded by technology, which tries to increase their quality of life and facilitate the daily activities. In addition, the continuous advancement in mobile computing makes it possible to obtain information about the context and also to react physically to it, in more innovative ways [1]. However, there are situations, where technology is difficult to be handled or people have lack of knowledge to use it. For these reasons, ambient intelligence (AmI) tries to adapt the technology to the people's needs by proposing three basic concepts: ubiquitous computing, ubiquitous communication, and intelligent user interfaces. In order to reach this objective, it is necessary to develop new frameworks and models to allow access to functionalities, regardless of time and location restrictions.

Dependence is a permanent situation in which a person needs important assistance from others in order to perform basic daily life activities, such as essential mobility, object and people recognition, or domestic tasks. There is an ever growing need to supply constant care and support to the disabled and elderly,

Manuscript received November 4, 2008; revised June 30, 2009. First published; current version published. This work was supported by the Spanish Ministry of Science and Technology project TIN2006-14630-C03-03.

J. M. Corchado is with the Faculty of Sciences and Biomedicine, Intelligent Systems and Educational Technology (BISITE) Research Group, University of Salamanca, Salamanca 37008, Spain and also with the Plymouth Marine Laboratory, Plymouth, U.K. (e-mail: corchado@usal.es).

J. Bajo is with the Pontifical University of Salamanca, Salamanca 37002, Spain (e-mail: jbajope@upsa.es).

D. I. Tapia is with the Biomedicine, Intelligent Systems and Educational Technology (BISITE) Research Group, University of Salamanca, Salamanca 37008, Spain (e-mail: dantetapia@usal.es).

A. Abraham is with the Machine Intelligence Research Laboratory (MIR), Scientific Network for Innovation and Research Excellence, Auburn, WA 98071 USA (e-mail: ajith.abraham@ieee.org).

Color versions of one or more of the figures in this paper are available online at http://ieeexplore.ieee.org.

Digital Object Identifier 10.1109/TITB.2009.2034369 and the drive to find more effective ways of providing such care has become a major challenge for the scientific community. The World Health Organization has determined that in the year 2025, there will be one billion people in the world over the age of 60 and twice as many by 2050 , with nearly $80 \%$ concentrated in developed countries [2]. In fact, people over 60 years old represent more than $21 \%$ of the European population [2], and people over 65 are the fastest growing segment of the population in the U.S.A. [3]. Furthermore, over $20 \%$ of those people over 85 have a limited capacity for independent living, requiring continuous monitoring and daily assistance [4]. The importance of developing new and more reliable ways of providing care and support for the elderly is underscored by this trend, and the creation of secure, unobtrusive, and adaptable environments for monitoring and optimizing healthcare will become vital [5].

This paper describes a telemonitoring system aimed at improving healthcare and assistance to dependent people at their homes. This system makes use of the Services laYers over Light PHysical devices (SYLPH) platform. SYLPH is based on a service-oriented rrchitecture (SOA) model for integrating heterogeneous wireless sensors networks (WSNs) into AmI systems. SYLPH focuses on distributing the systems' functionalities into independent functionalities (i.e., services). This model provides a flexible distribution of resources and facilitates the inclusion of new functionalities in highly dynamic environments. WSNs provide an infrastructure capable of supporting the distributed communication needed in highly dynamic environments, in this case, an AmI-based telemonitoring system for a dependency scenario, increasing mobility, flexibility, and efficiency, since resources can be accessed regardless their physical location [6].

The next section describes the motivation and explains why there is a need for developing a new telemonitoring system, using SYLPH. Then, the SYLPH platform is briefly described. Subsequently, the telemonitoring system is introduced, describing how SYLPH has been used to provide it with a robust wireless infrastructure. Finally, the results and conclusions are presented.

\section{Motivation AND PROBlem DesCRIPTION}

The emergence of AmI involves substantial changes in the design of functional architectures, since it is necessary to provide features, which enable a ubiquitous computing and communication, and also an intelligent interaction with users. This section discusses some of the most important problems of existent functional architectures, including their suitability for constructing intelligent environments according to the AmI paradigm. This section also presents the strengths and weaknesses of related 
developments, which integrate WSNs and analyzes the feasibility of a new alternative, using the SYLPH platform.

Excessive centralization of services negatively affects the systems' functionalities, overcharging, or limiting their capabilities. Classical functional architectures are characterized by trying to find modularity and a structure oriented to the system itself. Modern functional architectures like SOA, consider integration and performance aspects that must be taken into account when functionalities are created outside the system. An SOA-based system is a network of independent services, machines, the people who operate, affect, use, and govern those services, as well as the suppliers of equipment and personnel to these people and services [7]. The term service can be defined as a mechanism that facilitates the access to one or more functionalities (e.g., functions, network capabilities, etc.). The SOA model is aimed at the interoperability between different systems, distribution of resources, and the lack of dependency of programming languages [8]. Services are linked by means of standard communication protocols that must be used by applications in order to share resources in the services network [9]. The compatibility and management of messages that the services generate to provide their functionalities is an important and complex element in any of these approaches. A distributed architecture provides more flexible ways to move functions to where actions are needed, thus obtaining better responses at execution time, autonomy, services continuity, and superior levels of flexibility and scalability than centralized architectures [10]. Unfortunately, the difficulty in developing a distributed architecture is higher [11]. This way, it is necessary to have a more complex system analysis and design, which implies more time to reach the implementation stage.

AmI-based developments will require the use of several sensors and actuators strategically distributed in the environment. This provides the systems with context-aware capabilities in order to change its behavior automatically. It is possible to make a difference between sensor networks: wired and wireless. There are several technologies for creating wired sensors networks, such as X10, LonWorks, or KNX. However, wired networks are not as flexible as WSNs and require more infrastructural support [12], [13]. On the other hand, wireless technologies enable easier deployments than the wired ones, avoiding the need of wiring homes or hospitals and decreasing the costs, and drawbacks of the setup phase. The ZigBee standard allows operating in the frequency range belonging to the radio band known as industrial, scientific, and medical (ISM), especially in the $868 \mathrm{MHz}$ band in Europe, the $915 \mathrm{MHz}$ in the U.S.A., and the $2.4 \mathrm{GHz}$ in almost all over the world [14]. The underlying IEEE 802.15.4 standard is designed to work with low-power and limited computational resources nodes [15]. ZigBee incorporates additional network, application, and security layers over the 802.15.4 standard [16]. The ZigBee standard allows up to 65534 nodes connected in a star, tree, or mesh topology network. Another standard to deploy WSNs is Bluetooth. This standard allows multiple wireless personal Area networks (WPAN) and wireless body area networks (WBAN) applications for interconnecting mobile phones, earphones, personal computers, printers, etc. Bluetooth operates also in the ISM
$2.4 \mathrm{GHz}$ band. It allows creating star topology networks of up to eight devices in which one of them acts as master and the rest as slaves. Several Bluetooth networks can be interconnected by means of Bluetooth devices that belong simultaneously to two or more networks, creating more extensive networks [17].

Although there are plenty of options for creating WSNs, the main problem is the difficulty for integrating devices from different technologies in a single network [18]. In addition, the lack of a common architecture may lead to additional costs due to the necessity of deploying nontransparent interconnection elements between networks. Moreover, the developed elements (e.g., devices) are too dependent on the application to which they belong, thus complicating their reutilization. Some developments try to reach the devices integration by implementing middleware layers as reduced versions of virtual machines (e.g., Squawk Java virtual machine) [19]. These developments require devices with high computational power and large memory microcontrollers. For these reasons, there are needed more expensive devices with larger size or more costly miniaturization. These drawbacks are very important regarding WSNs, as it is essential to deploy applications with reduced resources and low infrastructural cost, especially in home care scenarios. The SYLPH platform integrates an SOA approach for facilitating the distribution and management of resources (i.e., services) into heterogeneous WSNs. There are several attempts to integrate WSNs and an SOA approach [20]-[25]. In SYLPH, unlike these approaches, services are directly embedded on the WSN nodes and can be invoked from other nodes in the same network or other network connected to the former one.

It is necessary to provide efficient solutions that allow building AmI environments for providing dependent people healthcare at their homes. One of the key aspects for the construction of these environments is obtaining context information through sensor networks. There are several healthcare developments for telemonitoring based on WSNs [26]-[28]. However, these developments do not take into account their integration with other systems and are difficult to be adapted to new situations. The use of SYLPH is proposed in order to face some of the issues found while integrating heterogeneous WSNs.

\section{SYLPH PLATFORM}

The SYLPH platform is a distributed architecture, which integrates an SOA approach over WSNs for building systems based on the AmI paradigm. The main objective is to distribute resources over multiple WSNs by modeling the functionalities as independent services. A service-oriented approach has been chosen because such architectures are asynchronous and nondependent on context (i.e., previous states of the system, which must not be confused with context-aware environments). Thus, devices working on them do not take continuously processing time and are free to do other tasks, or consume less energy.

SYLPH can be executed over multiple wireless devices independently of their microcontroller or the programming language they use. SYLPH works in a distributed way so that the application code does not have to reside almost completely on only central node. SYLPH allows the interconnection of several 


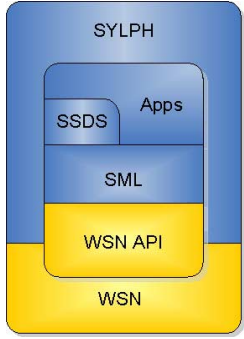

Fig. 1. SYLPH architecture layers.

networks from different wireless technologies, such as ZigBee or Bluetooth. Thus, a node designed over a specific technology can be connected to a node from a different technology. In this case, both WSNs are interconnected by means of a set of intermediate gateways connected to several wireless interfaces simultaneously. SYLPH allows applications to work in a distributed way and independent of the lower layers related to the WSNs formation (i.e., network layer), and the radio transmission amongst the nodes that conform them (i.e., data link and physical layers).

SYLPH implements an organization based on a stack of layers. Fig. 1 shows the different layers of SYLPH, which are added over the application layer of each WSN stack. The SYLPH message layer (SML) offers to the upper layers the possibility of sending asynchronous messages between two wireless devices through the SYLPH services protocol (SSP). The SSP is the internetworking protocol of the SYLPH platform. SSP has functionalities similar to those of the internet protocol (IP). That is, it allows sending packets of data from one node to another node regardless of the WSN to which each one belongs. The messages specify the origin and target nodes, and the service invocation in a SYLPH services definition language (SSDL) format. The SSDL describes the service itself and its parameters to be invoked. Applications can directly communicate between devices, using the SML layer or by means of the SYLPH services directory sublayer (SSDS) that uses in turn the mentioned SML layer. The SSDS offers functionalities related to the discovering of the services offered by the network nodes. A node that stores and maintains services tables is called SYLPH directory node (SDN).

The main components of SYLPH are now described.

\section{A. SYLPH Services}

The behavior of SYLPH is in essence similar to the one of any other SOA. However, SYLPH has several characteristics and functionalities that make it different from other models. Fig. 2 shows the basic operation of SYLPH.

First, a service registers itself on the SDN and informs its location in the network, the parameters it requires and the type of returned value after its execution. In order to do this, SSDL is used, which has been created to work with limited resources nodes. SSDL is the interface definition language (IDL) used by SYLPH. Distributed architectures use an IDL in order to enable communication between software components, regardless their programming language or hardware implementation. Un-

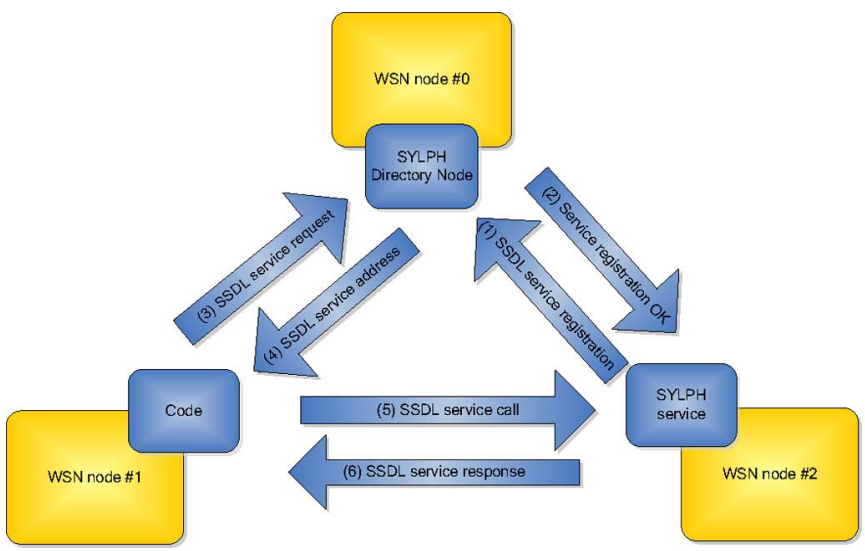

Fig. 2. SYLPH basic operation.

like other IDLs as WSDL, based on extensible markup language (XML) and used on web services [5]. SSDL use a few intermediate separating tags and its services descriptions are short binary data sequences.

The reason for these constraints is to reduce processing in the devices microcontrollers. Using a simple IDL allows, consequently, utilizing nodes with fewer resources, less power consumption, and lower cost. In most cases, it is enough with a few float point data for informing the status of a sensor. Thus, most service definitions require only a few bytes. SSDL considers the basic types of data (e.g., integer, float, or boolean), allowing more complex data structures as variable length arrays or character strings. In this way, SSDL is flexible enough to specify more complex services if required.

Once the service has been registered in the SDN, it can be invoked by any application by means of SYLPH. Both the SDN and the services can be stored in any node of the WSN or in other subsystem connected to the WSN. This system can be, for instance, a simple personal computer connected through an universal serial bus (USB) port to a wireless interface. Thus, developers decide, which nodes or subsystems will implement each part of the distributed application. Any node in the network can ask the SDN for the location of a determined service and its specification, using SSDL. This aspect is described in the following section.

\section{B. SYLPH Directory Nodes}

With the aim of the architecture to be the more distributed as possible, it is allowed to be more than one SDN in the same network, so that can exist redundancy or services organized in different directories. The SDN can be stored in a node of the network, with a memory external to the microcontroller if necessary, or be contained on a computationally higher machine connected to the WSN, as is the case of a data server or a personal computer with wireless connection.

Fig. 3 shows how a node can discover services in the network. For example, node 1 registers itself in the WSN by means of SYLPH. Then, it sends a broadcast message after connecting to the WSN searching for existing SDNs in the network. At this moment, only the node 0 is active, therefore, after receiving the 


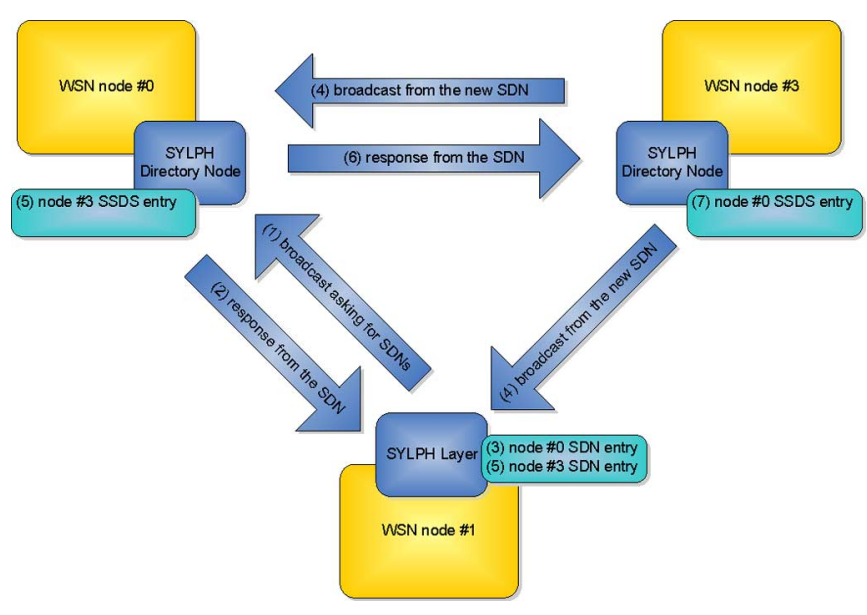

Fig. 3. SYLPH Directory Nodes.

broadcast message, it sends a message to the node 1 informing of its situation (i.e., SSP address) and its setup parameters. An example of a setup parameter is whether the SDN will inform periodically of its presence or if the nodeswill have to poll it. After this, the node 1 is able to communicate with the node 0 in order to obtain information about the possible services existing in the network. Later, the node 3 registers itself on the WSN. As it has SDN functionalities, it informs of this to the rest of the nodes by means of a broadcast message. The node 1 stores this information on its SSDS entries list and informs node 3 about its role as SDN. Any node in the network cannot only offer or invoke SYLPH services, but also includes SDN functionalities in order to provide services descriptions to other network nodes. SDNs include additional information about services, whose locations in the network maintain as, for example, a quality of service rate and a time stamp that represents the last time the SDN checked the service was available. An SDN can be configured to check the services, whose location stores or can be the services the responsible for broadcast themselves periodically.

\section{TELEMONITORING SySTEM}

This section describes the main features of a telemonitoring system aimed at improving healthcare of dependent people at their homes. The system makes use of several WSNs in order to gather context information in an automatic and ubiquitous way. Thus, the telemonitoring system enables an extensive integration of WSNs and provides a greater simplicity of deployment, thus optimizing the reutilization of the available resources in such networks.

Several functionalities are directly embedded on the WSN nodes and can be invoked from other nodes in the same network or other network connected to the former one by means of the SYLPH platform. SYLPH gateways are used in order to interconnect different heterogeneous WSNs. This way, SYLPH contemplates the possibility of connecting WSNs based on different radio and link technologies (e.g., ZigBee, Bluetooth, Wi$\mathrm{Fi}$, etc.), while other approaches do not. In addition, SYLPH focuses specially on devices with small resources in order to

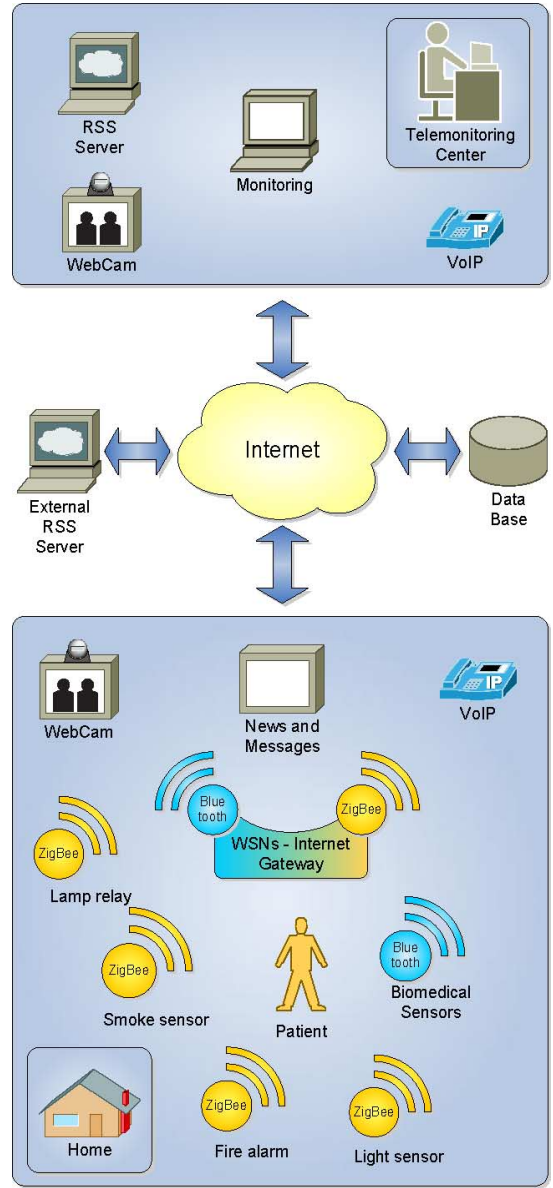

Fig. 4. Communication and infrastructure schema of the telemonitoring system.

save microcontrollers' computing time, memory data size, and energy consumption.

Biomedical sensors (e.g., electrocardiogram, blood pressure, body temperature, etc.) and automation sensors (e.g., building temperature, light, humidity, etc.) have significant differences, especially on how they collect data. Biomedical sensors obtain continuous information about vital signs, whose samples are important and should not be lost. On the other hand, automation sensors obtain information at a relatively lower frequency compared to biomedical sensors [29]. In addition, biomedical sensors should be smaller and easier to wear. It is necessary to interconnect several WSNs from different radio technologies in a telemonitoring scenario [27]. Having a compatible distributed platform for deploying healthcare applications over the different networks facilitates the developers' work and the integration of the heterogeneous devices.

Fig. 4 shows the basic communication and infrastructure schema of the telemonitoring system. A network of ZigBee devices has been designed to cover the home of each patient to be monitored. There is a ZigBee remote control carried by the monitored patient that incorporates a button, which can be pressed in case of remote assistance or urgent help. Moreover, there are a set of ZigBee sensors that obtain information about the home environment (e.g., light, smoke, temperature, doors' 
states, etc.) in which the user lives and that physically response to the changes (e.g., light dimmers, fire alarms, or door locks). Each of these ZigBee nodes includes a C8051F121 microcontroller and a CC2420 IEEE 802.15.4 radio frequency transceiver. There are also several Bluetooth biomedical sensors placed over the monitored patient's body. Biomedical sensors allow the system to acquire continuously data about the vital signs of the patient. In the telemonitoring system presented in this paper, each patient carries three different biomedical sensors: an ECG monitor, a respiration monitor (implemented by means of an air pressure sensor), and a micro-electro-mechanical systems (MEMS) triaxial accelerometer for detecting possible patient's falls. These Bluetooth nodes use a BlueCore4-Ext chip with a reduced instruction set computer (RISC) microcontroller with $48 \mathrm{kB}$ of RAM and $1024 \mathrm{kB}$ of external flash memory, and are compatible with the Bluetooth 2.0 standard. All these ZigBee and Bluetooth devices work as SYLPH nodes and can both offer and invoke functionalities (i.e., services) throughout the entire sensor network.

There is also a computer connected to a remote healthcare telemonitoring center via Internet. Alerts can be forwarded from the patients' homes to the caregivers in the remote center, allowing them to communicate with patients in order to check the possible incidences. These alerts can be, for instance, the detection of a patient's fall or a high smoke level in the patient's home. This computer acts as a ZigBee master node through a physical wireless interface (e.g., a ZigBee network adapter as a ZigBee USB dongle, or a ZigBee node connected through the computer's USB port). The computer is also the master node of a Bluetooth network formed by the biomedical sensors working as slave nodes. At the SYLPH level, the computer performs as a SYLPH gateway so that it connects both WSNs to each other.

If, for instance, the monitored patient falls over the floor, his fall detector gets from its Bluetooth accelerometer, a measurement higher than a previously specified threshold. This sensor (i.e., accelerometer) invokes a service stored in the WSNsInternet SYLPH gateway. Such service initiates a voice over Internet Protocol (VoIP) and webcam connection to be established between the telemonitoring center and the patient's home through Internet. This way, caregivers in the remote center can watch the patient's home and talk with him in order to verify the accident. As the telemonitoring center accesses directly to a patients database, caregivers can see his medical data and home address. If the accident is confirmed, the caregivers send an ambulance to his home.

Although this system is mainly focused on monitoring tasks, it also provides additional useful facilities to the patients and caregivers. For example, the remote center can consult really simple syndication (RSS) sources from external and internal web servers in order to obtain weather reports or entertainment options for patients and inform them of their scheduled medical staff visits. Such information is shown on a graphical user interface (GUI) on a display connected to the computer at home. This display is in fact a touch-sensitive screen, so that the interaction is easy and intuitive for patients. Moreover, the application includes home automation capabilities, so that a light sensor can make a lamp to be switched on or dimmed by means of the in-

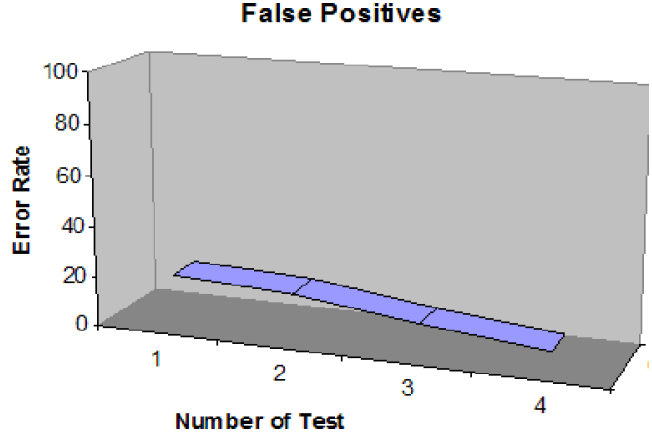

Fig. 5. False positives occurred during the test period of the alert subsystem.

vocation, or a certain service stored in a wireless actuator node connected to the relay or dimmer of the respective lamp.

\section{Results AND CONCLUSION}

The telemonitoring system presented in this paper improves security at home to dependents. It implements monitoring and alerting subsystems, as well as additional services to automatically react to emergency situations. We have done several test cases in order to evaluate the overall performance of the system, especially the management of emergency situations. The tests, which involved 13 patients and six caregivers, allowed us to evaluate the system. Specifically, the results of the SYLPH system were studied over a period of four weeks.

We have evaluated our approach in terms of the four main objectives, defined for WSN applied in healthcare developments: minimize error rates, conduct diagnosis with real time patient data, improve efficiency, and reduce costs [26]. We have collected data before and after the implantation of the telemonitoring system, with data collected from Feb. 2009 to Mar. 2009. The system was adopted on Mar. 1, 2009.

Firstly, our approach provides the system with a higher ability to recover from errors. Fig. 5 shows the percentage of error that occurred in the alert subsystem during the tests. The initial tests showed an error rate above $19 \%$. This percentage was primarily due to errors in the use of the system by the caregivers. After the third test, the error rate was reduced to $11 \%$. From this point the error rate remained stable at $4 \%$. This error could be reduced with a higher level of training in the use of the telemonitoring system.

Secondly, we have worked with real-time data from the patients. These data have not been used for diagnosis, but to assess the need for providing healthcare to the patients. In this sense, SYLPH reaches this goal because it can work with real-time data. However, it is necessary to include a reasoning mechanism in order to facilitate the decision making for diagnosis.

Finally, the efficiency of the telemonitoring system has been enhanced and the infrastructure costs has been reduced. SYLPH allowed us to determine the time needed to respond to alerts generated by the users or the sensors. In order to generalize reactions to common situations, the system does not only take response time into account, but also the time elapsed between alerts and the user's profile and reliability. The telemonitoring 
system allows a reduction of the response time to incidents (i.e., alerts). When comparing data before and after the implantation of the telemonitoring system, we concluded that telemonitoring system provides an average response time to incidents of $8 \mathrm{~min}$, which reduces the time employed by the caregivers in a $7 \%$. Moreover, the average of assisted incidents per day was 12 , providing a reduction of $11 \%$. This reduction can be directly associated to the improvement in the detection of false positives. Besides, the time employed by the medical staff to attend an alert has been notably reduced.

SYLPH allows wireless sensor devices from different radio technologies to work together in a distributed way. These sensor devices do not have to be provided with large memory chips or fast microprocessors. The SYLPH model allows the possibility of adding or removing components at execution time. This way, it is possible to add a new automation sensor in the patient's home at execution time with no need of reprogram or redeploy the system. If, for example, it is decided to add a new smoke sensor in the patient's home, the technician will just have to fix it to the ceiling and supplies it with electrical power (e.g., a battery or the electric power network). The ZigBee node will join automatically to the ZigBee automation network, and its SYLPH layers will look for SDNs and services in the SYLPH network and register its own services on them. This way, if the new smoke sensor detects a dangerous smoke level, it will automatically invoke the smoke alarm service in the alarm nodes in the home and the WSNs-Internet SYLPH gateway.

The telemonitoring system's architecture goes a step ahead in designing systems for home care by offering features that make it easily adaptable to any pervasive environment. Unlike other telemonitoring systems as [26], [27], or [28], the presented system allows to integrate heterogeneous sensor networks from different technologies, even wired ones. Therefore, it is possible to join automation and biomedical sensors in the same telemonitoring system, using the technology that better fits each sensor's data characteristics. Furthermore, as result of the dynamic features of the SYLPH platform, the presented system can be easily enhanced with new sensors without be redeployed, and it can be adapted to new scenarios as even whole medical centers with many patients, whilst other cited approaches cannot.

Although the initial results are promising, the telemonitoring systems still requires some improvements. Our future work focuses on finding a modeling facility to provide learning and decision-making capabilities to the system. The objective is to facilitate the integration of SYLPH within intelligent systems aimed at facilitating automatic decision support mechanisms for healthcare. In this sense, SYLPH can provide an excellent framework to obtain real-time data. We believe that the agents' technology can result adequate to establish the link between SYLPH and intelligent systems. Another important issue is to improve the privacy and security requirements, and we have begun to explore different alternatives.

\section{REFERENCES}

[1] G. T. Jayaputera, A. Zaslavsky, and S. W. Loke, "Enabling run-time composition and support for heterogeneous pervasive multiagent systems," $J$. Syst. Softw., vol. 80, pp. 2039-2062, 2007.
[2] W. H. Organization, Global Age-Friendly Cities. Geneva, Switzerland: World Health Organization, 2007.

[3] R. N. Anderson, "A method for constructing complete annual U.S. life tables," Vital Health Statist. Ser, vol. 129, pp. 1-28, 2000.

[4] P. Erickson, Years of Healthy Life. Hyattsville, MD: U.S. Dept. of Health and Human Services, Public Health Service, Centers for Disease Control and Prevention, National Center for Health Statistics, 1995.

[5] J. M. Corchado, J. Bajo, Y. D. Paz, and D. I. Tapia, "Intelligent environment for monitoring Alzheimer patients, agent technology for healthcare," Decis. Support Syst., vol. 44, pp. 382-396, 2008.

[6] M. Gast and M. S. Gast, 802.11 Wireless Networks: The Definitive Guide. Sebastopol, CA: O'Reilly, 2002.

[7] OASIS Open, Reference Model for Service Oriented Architecture 1.0, OASIS Standard, Oct. 2006.

[8] E. Cerami, Web Services Essentials: Distributed Applications with XMLRPC, Soap, UDDI and Wsdl. Sebastopol, CA: O'Reilly Media, 2002.

[9] L. Ardissono, G. Petrone, and M. Segnan, "A conversational approach to the interaction with web services," Comput. Intell., vol. 20, pp. 693-709, 2004.

[10] L. Camarinha-Matos and H. Afsarmanesh, "A comprehensive modeling framework for collaborative networked organizations," J. Intell. Manuf., vol. 18, pp. 529-542, Oct. 2007.

[11] P. Rigole, T. Holvoet, and Y. Berbers, "Using Jini to integrate home automation in a distributed software-system," in Distributed Communities on the Web. Berlin, Germany: Springer-Verlag, 2002, pp. 185-232.

[12] A. Ahmed, J. Ali, A. Raza, and G. Abbas, "Wired vs wireless deployment support for wireless sensor networks," in Proc. 2006 IEEE Region 10 Conf., TENCON, pp. 1-3.

[13] M.-h. Lee and H. Yoe, "Comparative analysis and design of wired and wireless integrated networks for wireless sensor networks," in Proc. 5th ACIS Int. Conf. Softw. Eng. Res., Manage. Appl. (SERA 2007), pp. 518522.

[14] Y. Huang and A. Pang, "A comprehensive study of low-power operation in IEEE 802.15.4," in Proc. 10th ACM Symp. Modeling, Anal., Simul. Wireless Mobile Syst., Chania, Crete Island, Greece: ACM, 2007, pp. 405408.

[15] C. Singh, A. Kumar, and P. Ameer, "Performance evaluation of an IEEE 802.15.4 sensor network with a star topology," Wireless Netw., vol. 14, pp. 543-568, 2008.

[16] P. Baronti, P. Pillai, V. W. C. Chook, S. Chessa, A. Gotta, and Y. F. Hu, "Wireless sensor networks: A survey on the state of the art and the 802.15.4 and ZigBee standards," Comput. Commun., vol. 30, pp. 1655-1695, 2007.

[17] M. Ilyas, The Handbook of Ad hoc Wireless Networks. Boca Raton, FL: CRC Press, 2002.

[18] S. Lei, H. Xu, W. Xiaoling, Z. Lin, J. Cho, and S. Lee, "VIP bridge: Integrating several sensor networks into one virtual sensor network," in Proc. Int. Conf. Internet Surveill. Prot. (ICISP 2006), p. 2.

[19] D. Simon and C. Cifuentes, "The squawk virtual machine: Java ${ }^{\mathrm{TM}}$ on the bare metal," in Proc. Companion 20th Annu. ACM SIGPLAN Conf. Object-Oriented Program., Syst., Lang., Appl., San Diego, CA: ACM, 2005, pp. 150-151.

[20] E. Meshkova, J. Riihijärvi, F. Oldewurtel, C. Jardak, and P. Mähönen, "Service-oriented design methodology for wireless sensor networks: A view through case studies," in Proc. IEEE Int. Conf. Sens. Netw., Ubiquitous, Trustworthy Comput. (sutc), Washington, DC: IEEE Computer Society, 2008, vol. 00, pp. 146-153.

[21] R. Moeller and A. Sleman, "Wireless networking services for implementation of ambient intelligence at home," in Proc. 7th Int. Caribbean Conf. Devices, Circuits Syst. (ICCDCS 2008), pp. 1-5.

[22] J. M. Prinsloo, C. L. Schulz, D. G. Kourie, W. H. M. Theunissen, T. Strauss, R. V. D. Heever, and S. Grobbelaar, "A service oriented architecture for wireless sensor and actor network applications," in Proc. 2006 Annu. Res. Conf. South Afri. Inst. Comput. Sci. Inf. Technol. IT Res. Develop. Countries, Somerset West, South Africa, South African Institute for Computer Scientists and Information Technologists, pp. 145-154.

[23] A. Sleman and R. Moeller, "Integration of wireless sensor network services into other home and industrial networks; using device profile for web services (DPWS)," in Proc. 3rd Int. Conf. Inf. Commun. Technol.: From Theory Appl. (ICTTA 2008), pp. 1-5.

[24] E. Song and K. Lee, "Smart transducer web services based on the IEEE 1451.0 standard," in Proc. IEEE Instrum. Meas. Technol. Conf. (IMTC 2007), pp. 1-6.

[25] E. Song and K. Lee, "STWS: A unified web service for IEEE 1451 smart transducers," IEEE Trans. Instrum. Meas., vol. 57, no. 8, pp. 1749-1756, Aug. 2008. 
[26] L. Fass, "Patient-centric healthcare," in Proc. 3rd Inst. Eng. Technol. Int. Conf. Med. Electr. Devices Technol. (MEDTECH 2007), pp. 77-109.

[27] A. D. Jurik and A. C. Weaver, "Remote medical monitoring," Computer, vol. 41, pp. 96-99, 2008.

[28] U. Varshney, "Improving wireless health monitoring using incentive-based router cooperation," Computer, vol. 41, pp. 56-62, 2008.

[29] J. Sarangapani, Wireless Ad hoc and Sensor Networks: Protocols, Performance, and Control. Boca Raton, FL: CRC Press, 2007.

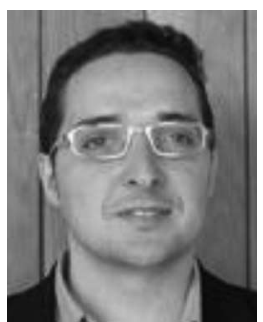

Juan M. Corchado received the Ph.D. degree in computer science from the University of Salamanca, Salamanca, Spain, and the Ph.D. degree in artificial intelligence from the University of Paisley, Paisley, U.K.

He is the Dean of the Faculty of Computer Science and an Associate Professor with the University of Salamanca, where he directs the Biomedicine, Intelligent Systems and Educational Technology (BISITE) Research Group (http://bisite.usal.es) and the M.Sc. programs in computer science. Since 1993, he has also been a Research Collaborator with the Plymouth Marine Laboratory, Plymouth, U.K.. His current research interests include agents and multiagent systems, ambient intelligence, case-based reasoning, and real-time forecasting.

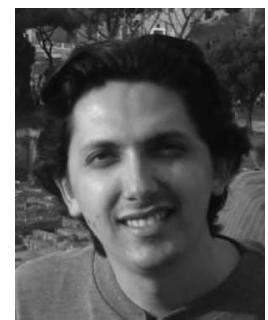

Dante I. Tapia received the Ph.D. degree in computer science from the University of Salamanca, Salamanca, Spain.

$\mathrm{He}$ is a Researcher with the Biomedicine, Intelligent Systems and Educational Technology (BISITE) Research Group, University of Salamanca. His current research interests include multiagent systems, ubiquitous computing, and distributed architectures.

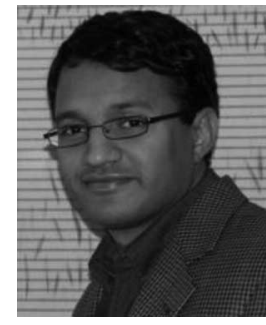

Ajith Abraham received the Ph.D. degree in computer science from Monash University, Melbourne, Victoria, Australia.

$\mathrm{He}$ is the Director of Machine Intelligence Research Laboratories (MIR Laboratories), Scientific Network for Innovation and Research Excellence, Auburn, WA. His current research interests include computational intelligence, focusing on applications, such as network security, web services, web intelligence, and data mining.He is author or coauthor of more than 500 publications.

Dr. Abraham is the Cochair of the IEEE TRANSACTIONS ON SYSTEMS, MAN, AND CYBERNETICS Technical Committee on Soft Computing.

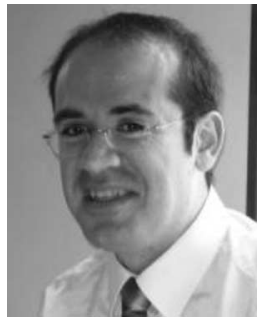

Javier Bajo received the Ph.D. degree in computer science from the University of Salamanca, Salamanca, Spain.

$\mathrm{He}$ is an Associate Professor with the Pontifical University of Salamanca. His current research interests include multiagent systems, decision support systems, and intelligent environments. 